\title{
Un peu plus de lumière sur les lipofuscinoses céroïdes
}

Les lipofuscinoses céroïdes (CLN), maladies neurodégénératives héréditaires, sont caractérisées par une accumulation de lipopigments autofluorescents, principalement dans les cellules nerveuses. Elles se manifestent par une dégradation psychique, des crises d'épilepsie et une perte progressive de la vision. Classées initialement d'après le mode d'apparition des signes cliniques et l'aspect des dépôts lysosomaux, elles sont progressivement répertoriées grâce à l'isolement des gènes en cause. Six locus ont été identifiés, mais il est certain que la liste n'est pas close (Tableau I).

Les mécanismes biochimiques à l'origine de ces CLN commencent à être élucidés sans que l'on puisse expliquer la cause de l'accumulation de la sous-unité c de l'ATPase mitochon- driale, un des composants majeurs des dépôts de lipopigments. Toutefois des travaux récents ont permis de progresser dans la connaissance des deux formes les plus fréquentes de CLN : LINCL (late infantile neuronal ceroid lipofuscinosis) et JNCL (juvenile neuronal ceroid lipofuscinosis).

Alors que la forme infantile (INCL) est due à l'absence d'une enzyme lysosomale, la palmito-protéine thioestérase, codée par le gène CLN1 [1], la forme infantile tardive (LINCL) comporte deux variantes : la variante finlandaise, due à des mutations de CLN5, qui code pour une protéine de la membrane dont la fonction est encore inconnue [2], et la forme classique dont le gène en cause, CLN2, code pour une protéase lysosomale [3]. Une comparaison avec la séquence de l'enzyme tripeptidyl peptidase I (TPP I) suggère qu'il s'agit bien de la même protéase. Reste à savoir si l'accumulation de l'ATPase mitochondriale est la conséquence directe ou indirecte de la déficience en CLN2.

\section{CLN2 ou TPP1, analyse des mutations}

Un groupe international vient de rendre compte d'une étude génétique portant sur 74 familles de LINCL de forme classique européennes [4]. Vingt-quatre mutations de CLN2 ont été identifiées, dont deux, une mutation non sens et une mutation modifiant un site d'épissage, sont situées dans le même segment de l'exon 6 et trouvées chez plus de $60 \%$ des malades originaires

\section{Tableau I}

PRINCIPALES LIPOFUSCINOSES CEROÏDES, DANS LEUR CLASSIFICATION ACTUELLE

\begin{tabular}{|c|c|c|c|c|c|}
\hline Maladie & Gène & Transmission & Localisation & Produit & Fonction \\
\hline $\begin{array}{l}\text { ICLN } \\
\text { Maladie de Santavuori-Haltia } \\
\text { (forme finlandaise) }\end{array}$ & CLN1 & RA & $1 p 32$ & $\begin{array}{l}\text { palmitoyl protéine } \\
\text { thioestérase ou PPT }\end{array}$ & \\
\hline $\begin{array}{l}\text { LINLC } \\
\text { idiotie amaurotique infantile tardive } \\
\text { Maladie de Jansky-Bielschowsky }\end{array}$ & CLN2 & RA & $11 \mathrm{p} 15$ & $\begin{array}{c}\text { tripeptidyl peptidase acid } \\
\text { insensible à la peptasine } \\
\text { ou TPPI }\end{array}$ & \\
\hline $\begin{array}{l}\text { JNVCL } \\
\text { Maladie de Spielmeyer-Vogt-Batten }\end{array}$ & CLN3 & RA & $16 \mathrm{p} 12$ & $\begin{array}{c}\text { protéine } \\
\text { transmembranaire }\end{array}$ & $\begin{array}{l}\text { Homéostasie } \\
\text { du pH } \\
\text { des vacuoles }\end{array}$ \\
\hline $\begin{array}{l}\text { NLC forme adulte } \\
\text { Maladie de Kufs }\end{array}$ & CLN4 & $\begin{array}{l}\text { RA } \\
\text { ou } \\
\text { DA }\end{array}$ & $? ?$ & $? ?$ & \\
\hline $\begin{array}{l}\text { LINLC } \\
\text { Variante finlandaise }\end{array}$ & CLN5 & RA & $13 q 21-32$ & $\begin{array}{c}\text { protéine } \\
\text { transmembranaire }\end{array}$ & \\
\hline LINCL & CLN6 & RA & $15 q 21-23$ & $? ?$ & \\
\hline
\end{tabular}

RA: récessif autosomique. DA: dominant autosomique; PPT: palmytoil protéine thioestérase; TPPI: tripeptidyl peptidase I. 
du Nord de l'Europe. Pour les malades de l'Est, du Centre et du Sud de l'Europe, les mutations sont beaucoup plus variées (deux mutations sont pour l'instant exclusivement italiennes), et sont disséminées tout le long de la séquence codante de CLN2. Aucune corrélation entre génotype et phénotype n'a pu être dégagée. Il est intéressant de noter qu'une mesure rapide de l'activité CLN2 protéase, utilisable sur de nombreux tissus, permet une sélection des malades. En effet, chez les patients dont l'activité CLN2 protéase est intacte, aucune mutation n'a été décelée. Chez quelques malades considérés comme atteints de la forme juvénile (ou maladie de Batten), mais dont les inclusions étaient atypiques, une mutation (substitution Arg $\rightarrow$ His) a été identifiée.

\section{CLN3 et BTN1 : histoires parallèles}

Le «Consortium international de la maladie de Batten » a réussi à isoler le gène en cause, $C L N 3$, en $1995(\mathrm{~m} / \mathrm{s}$ 1995, $\left.n^{\circ} 12, p .1752\right)$. Bien que le gène murin homologue ait été isolé rapidement par la suite [5], on ignorait jusqu'à présent le rôle intracellulaire de la protéine CLN3. Récemment, en fusionnant CLN3 avec GFP (green fluorescent protein) et en transfectant diverses lignées cellulaires avec cette protéine de fusion, on a pu observer que CLN3 était localisée initialement dans l'appareil de Golgi [6, 7]. Elle serait ensuite transportée vers les lysosomes par le système transgolgien [8]. Sa caractérisation biochimique, ainsi que l'observation au microscope confocal montre que CLN3, appelée désormais aussi «battenine», est une protéine de la membrane lysosomale dont la fonction protéolytique s'exercerait dans les compartiments acides de la cellule [9]. Pendant que plusieurs équipes de chercheurs poursuivaient in vitro l'étude de la localisation subcellulaire et la caractérisation biochimique de CLN3, une cascade de travaux de deux spécialistes américains des levures fournissait de précieux renseignements sur CLN3. Les étapes principales, pour faire bref, furent les suivantes: (1) isolement du gène orthologue de CLN3 chez Saccharomyces cerevisiae, BTN1 (pour Bat- ten), dont le produit possède $39 \%$ d'identité et $59 \%$ de similitude avec CLN3 [10] ; (2) création de souches dépourvues de $B T N 1$, dont la multiplication est normale, mais qui sont résistantes à l'ANP, une substance chimique inhibant le développement des souches BTN1+ ; (3) mise en évidence de l'acidification rapide du milieu de culture en début de multiplication des souches BTN1-D, cette acidification conférant la résistance à l'ANP; (4) complémentation par addition du gène $C L N 3$, démontrant l'identité de fonction des deux protéines. A ce stade, les chercheurs se trouvaient donc en possession d'un modèle pour la maladie de Batten. En complémentant avec des gènes $C L N 3$ mutés provenant de malades, ils purent constater une corrélation inverse entre le degré de gravité de la maladie et le degré de correction du phénotype de résistance à l'ANP dans les souches BTN1-D, ainsi que une répercussion identique sur le degré de résistance à l'ANP des mêmes substitutions d'acides aminés dans les protéines BTN1 et CLN3 [11]. Enfin, dans les souches BTN1-D, la délétion de BTN1 entraîne une augmentation de l'expression de deux autres gènes : HSP3O (pour heat shock protein) qui code pour une protéine membranaire diminuant l'ATPase de la membrane plasmatique, et BTN2 qui code pour la protéine $\mathrm{HOOK}$, présente, entre autres, chez l'homme et la drosophile [12]. Chez cet insecte, on a pu montrer que la protéine HOOK est impliquée dans l'endocytose des ligands transmembranaires, un processus sous la dépendance de l'acidification des vésicules [13]. Or, BTN1 est localisée dans les vacuoles et doit intervenir en les acidifiant. Les lysosomes humains sont analogues aux vacuoles de S. cerevisiae. On suppose donc que l'abaissement du $\mathrm{pH}$ des lysosomes pourrait, soit diminuer l'activité de certaines protéases, soit entraîner l'accumulation et l'agrégation de protéines cibles. Des travaux chez la souris ont montré que les neurones étaient particulièrement sensibles aux troubles de régulation du $\mathrm{pH}$. Il est donc logique de supposer que la dégénérescence neuronale de la maladie de Batten est la conséquence d'une anomalie de régulation du $\mathrm{pH}$ due à l'absence fonctionnelle de la protéine de membrane lysosomale CLN3.

Il s'agit donc d'une première étape dans la compréhension des mécanismes conduisant à l'accumulation des lipopigments autofluorescents et à la mort neuronale, mais elle est prometteuse. La découverte des autres gènes CLN de ces maladies gravissimes jadis appelées idioties amaurotiques familiales ne saurait tarder.

\section{Simone Gilgenkrantz \\ 9, rue Basse, 54330 Clerey-sur-Brenon, France.}

1. Camp LA, Hoffmann SL. Purification and properties of palmitoyl-protein thioesterase tha cleaves palmitate from H-Ras J Biol Chem 1993; 268 : 22566-74.

2. Sleat DE, Donnely RJ, Lackland H, et al. Association of mutations in a lysosomal protein with classical late-infantile neuronal ceroid lipofuscinosis. Science 1997; 277: 1802-5.

3. Savikoski M, Klockars T, Holmberg V, Santavuori P, Lander ES, Peltonen L. CLN5, a novel gene encoding a putative transmembrane protein mutated in Finnish variant late infantile neuronal ceroid lipofuscinosis. Nat Genet 1998; 19: 286-88.

4. Sleat DE, Gin RM, Sohar I, et al. Mutational analysis of the defective protease in classic lateinfantile neuronal ceroid lipofuscinosis, a neurodegenerative lysosomal storage disorder. Am J Hum Genet 1999; 64 : 1511-23.

5. Lee RL, Johnson KR, Lerner TJ. Isolation and chromosomal mapping of a mouse homolog of the Batten disease gene, CLN3. Genomics 1996; 35 : 617-9. 6. Kremmidiotis G, Lensink IL, Bilton RL et al. The Batten disease gene product (CLN3p) is a Golgi integral membrane protein. Hum Mol Genet 1999; 8: 523-31.

7. Michalewski MP, Kaczmarski W, Golabek AA, Kida E, Kaczmarski A, Wisniewski KE. Post-translational modification of CLN3 protein and its possible functional implication. Mol Genet Metab $1999 ; 66: 272-6$

8. Golabeck AA, Kaczmarski W, Kida EK, Michaliewski MP, Winiewski KE. Expression studies of CLN3 protein (Battenin) in fusion with the green fluorescent protein in mammalian cells in vitro. Mol Genet Metab 1999; 66 : 277-82.

9. Kida E, Kaczmarski W, Golabek AA, Kaczmarski A, Michaliewski MP, Wisniewsli KE. Analysis of intranuclear distribution and trafficking of the CLN3 protein in fusion with the green fluorescent protein in vitro. Mol Genet Metab 1999; 66: 265-71. 10. Pearce DA, Sherman F. BTN1, a yeast gene corresponding to the human gene responsible fot Batten's disease is not essential for viability, mitochondrial function, or degradation of mitochondrial ATPsynthase. Yeast 1997; 13: 691-7.

11. Pearce DA, Sherman F. A yeast model for the study of Batten disease. Proc Natl Acad Sci USA $1998 ; 95$ 6915-8.

11. Pearce DA, Ferea T, Nosel SA, Das B, Sherman F. Action of BTN1, the yeast orthologue of the gene mutated in Batten disease. Nat Genet 1999 ; 22 : 55-8.

12. Kramer H, Philstry M. Mutations in the drosophila hook gene inhibit endocytosis of the boss transmembrane ligand into multivesicular bodies. J Cell Biol 1996; 133: 1205. 